\section{Shiitake-Polysaccharide als Antiallergika?}

In Pilzzellwänden finden sich $\beta$-1,3-Glukane, denen starke immunmodulierende Effekte zugeschrieben werden. Eine japanische Forschergruppe untersuchte jetzt den Effekt von $\beta$-1,3-Glukan auf die Symptomatik von Patienten mit einer Zedernpollenallergie.

D as bekannteste $\beta$-1,3-Glukan ist Lentinan, das aus Shiitake-Pilzen gewonnen wird. Lentinan ist zugelassen zur immunmodulatorischen Behandlung von Patienten mit inoperablem Magenkarzinom. Wichtige Wirkmechanismen sind die Aktivierung von MakrophagenSubpopulationen und eine damit verbundene TH1-Antwort. Glukane sind relativ große Moleküle, die sich in Wasser nur mit einer Partikelgröße von 100 bis $200 \mu \mathrm{m}$ dispergieren lassen und im Magen-Darm-Trakt nicht resorbiert werden. Deshalb war bisher nur eine intravenöse Gabe therapeutisch erfolgreich. Superfein in Wasser dispergierte Glukane sind dagegen auch oral aktiv.

In einer randomisierten doppelblinden Pilotstudie erhielten Patienten mit Rhinokonjunktivitis aufgrund der in Japan häufigen Zedernpollenallergie entweder feindisperses $\beta$-1,3-Glukan $(\mathrm{n}=$ 30) oder nicht dispergiertes $\beta-1,3$-Glu- kan als Plazebo $(\mathrm{n}=30)$. Die Glukane stammten aus Shiitake-Pilzen und wurden in einer Dosierung von $15 \mathrm{mg}$ über acht Wochen gegeben. Der Therapiebeginn lag bei einem Teil der Patienten vor Einsetzen des Pollenflugs, bei den Übrigen während der Saison.

Im Vergleich zum nicht resorbierbaren Plazebo-Glukan führte die Ein-

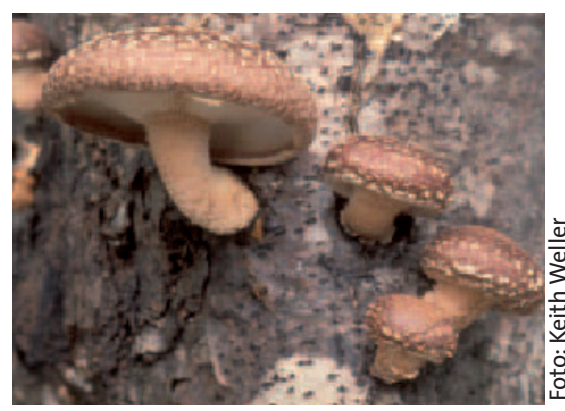

Schmecken nicht nur lecker, sondern lindern auch Heuschnupfenbeschwerden: Shiitake-Pilze (Lentinus edodes). nahme von feindispersem Glukan zu einer deutlichen Symptomreduktion innerhalb von ein bis zwei Wochen nach Beginn der Einnahme. Bei Einnahme vor Beginn der Pollenflugsaison wirkten die Polysaccharide auch präventiv. Der saisonbedingte Anstieg des allergenspezifischen IgE und des Gesamt-IgE im Serum war unter dem feindispersen Polysaccharid im Vergleich zu Plazebo reduziert. Der klinische Effekt korrelierte mit der Bindungsfähigkeit der Monozyten der Patienten für $\beta-1,3$-Glukan. Dies spricht dafür, dass die beobachtete Wirkung vermutlich über Glucan-aktivierte Makrophagen vermittelt wird, die antiinflammatorische Mediatoren wie TGF$\alpha$ und IL-10 freisetzen und über die Produktion von IL-12 zusätzlich eine TH1Antwort induzieren.

Fazit: In dieser Studie reduzierte die orale Einnahme von feindispersem $\beta-1,3-$ Glucan die Symptomatik von Patienten mit einer Zedernpollenallergie, auch eine präventive Gabe war wirksam.

$b k$

Yamada J et al. Alleviation of seasonal allergic symptoms with superfine beta-1,3glucan: a randomized study. J Allergy Clin Immunol 2007; 119: 1119-26

\title{
Antihistaminika im direkten Vergleich
}

\section{Eine Möglichkeit, die Effektivität von Antihistaminika zu ver- gleichen, ist es, deren Einfluss auf die Hautreaktivität bei einem Pricktest mit Histamin zu quantifizieren. Diese Untersuchung wurde jetzt erstmals für die beiden modernen $\mathrm{H}_{1}$-Rezeptor- antagonisten Fexofenadin und Desloratadin durchgeführt.}

$\mathrm{K}$ alifornische Forscher rekrutierten für ihre Cross-over-Studie 54 gesunde Probanden. Diese erhielten oral entweder $180 \mathrm{mg}$ Fexofenadin-HCl, 5 mg Desloratadin oder Plazebo. Dann erfolgte am Unterarm die Untersuchung mit Histamin im Pricktest, in den ersten zwölf Stunden nach Antihistaminikaeinnahme einmal pro Stunde sowie nach 23 und 24 Stunden. Die Erytheme und Quaddeln wurden vermessen und dokumentiert. Nach einer Auswaschperiode von zehn bis $28 \mathrm{Ta}$ gen erfolgte die erneute Testung mit einer der anderen beiden Substanzen im Cross-over-Design.

In der Studie unterdrückte Fexofenadin zwei bis sechs Stunden nach Einnahme die Erythembildung signifikant stärker als Desloratadin. Auch die Quaddelbildung wurde durch Fexofenadin im Vergleich zu Desloratadin in den Zeiträumen zwei bis vier Stunden nach Einnahme sowie sechs bis neun und zwölf Stunden danach signifikant stärker reduziert. Im Vergleich zu Plazebo war die Quaddelbildung durch Fexofenadin im Zeitraum zwischen zwei und zwölf
Stunden nach Einnahme, die Erythemgröße zwischen zwei und 24 Stunden nach Einnahme des Antihistaminikums signifikant reduziert. Auch Desloratadin reduzierte gegenüber Plazebo signifikant die Erythembildung, aber nur in den Zeitfenstern sechs bis zehn bzw. zwölf und 24 Stunden nach Einnahme. Die Quaddelbildung war durch Desloratadin im Vergleich zu Plazebo nur nach zehn Stunden signifikant reduziert.

Fazit: Fexofenadin zeigte in dieser Untersuchung im Vergleich zu Desloratadin einen rascheren Wirkungseintritt und eine höhere Effektivität in Bezug auf die Reduktion von Quaddel- und Erythembildung nach Histaminpricktest. $\quad f k$

Meltzer EO et al. Efficacy of fexofenadine versus desloratadine in suppressing histamine-induced wheal and flare. Allergy Asthma Proc 2007; 28: 67-73 